\title{
Stability and Accuracy of 3D Neutron Transport Simulations using the 2D/1D Method in MPACT is
}

\author{
Benjamin Collins $^{\mathrm{a}, 1, *}$, Shane Stimpson ${ }^{\mathrm{a}, 1}$, Blake W. Kelley ${ }^{\mathrm{b}}$, Mitchell T.H. \\ Young $^{\mathrm{b}}$, Brendan Kochunas ${ }^{\mathrm{b}}$, Aaron Graham ${ }^{\mathrm{b}}$, Edward W. Larsen ${ }^{\mathrm{b}}$, \\ Thomas Downar ${ }^{\mathrm{b}}$, Andrew Godfrey ${ }^{\mathrm{a}}$ \\ ${ }^{a}$ Oak Ridge National Laboratory, One Bethel Valley Rd. Oak Ridge, TN 37831 \\ ${ }^{b}$ Department of Nuclear Engineering and Radiological Sciences, University of Michigan, \\ Ann Arbor, Michigan 48109
}

\begin{abstract}
A consistent "2D/1D" neutron transport method is derived from the 3D Boltzmann transport equation, to calculate fuel-pin-resolved neutron fluxes for realistic full-core Pressurized Water Reactor (PWR) problems. The 2D/1D method employs the Method of Characteristics to discretize the radial variables and a lower order transport solution to discretize the axial variable. This paper describes the theory of the 2D/1D method and its implementation in the MPACT code, which has become the whole-core deterministic neutron transport solver for the Consortium for Advanced Simulations of Light Wa-
\end{abstract}

\footnotetext{
This manuscript has been authored by UT-Battelle, LLC under Contract No. DEAC05-00OR22725 with the U.S. Department of Energy. The United States Government retains and the publisher, by accepting the article for publication, acknowledges that the United States Government retains a non-exclusive, paid-up, irrevocable, world-wide license to publish or reproduce the published form of this manuscript, or allow others to do so, for United States Government purposes. The Department of Energy will provide public access to these results of federally sponsored research in accordance with the DOE Public Access Plan (http://energy.gov/downloads/doe-public-access-plan).

${ }^{*}$ Corresponding Author

Email addresses: collinsbs@ornl.gov (Benjamin Collins), stimpsonsg@ornl.gov (Shane Stimpson), kelleybl@umich.edu (Blake W. Kelley), youngmit@umich.edu (Mitchell T.H. Young), bkochuna@umich.edu (Brendan Kochunas), aarograh@umich.edu (Aaron Graham), edlarsen@umich.edu (Edward W. Larsen), downar@umich.edu (Thomas Downar), godfreyat@ornl.gov (Andrew Godfrey)

${ }^{1} \mathrm{~A}$ large component of this work was performed while the author was at the University of Michigan.
}

Preprint submitted to Journal of Computational Physics

August 16, 2016

(C) 2016. This manuscript version is made available under the Elsevier user license http://www.elsevier.com/open-access/userlicense/1.0/ 
ter Reactors (CASL) core simulator VERA-CS. Several applications have been performed on both leadership-class and industry-class computing clusters. Results are presented for whole-core solutions of the Watts Bar Nuclear Power Station Unit 1 and compared to both continuous-energy Monte Carlo results and plant data.

Keywords: 2D/1D Method, MOC, MPACT

\section{Introduction}

Significant advances in multi-core, multi-node computing clusters over the past several years have enabled the development of practical numerical methods for solving the Boltzmann Transport Equation (BTE) to calculate fuel-pin-resolved neutron fluxes in full 3D nuclear reactor cores. Historically, the nuclear reactor industry has relied upon a two-step procedure to solve for full-core power distributions - by using pre-generated few-group cross sections, homogenized over a fuel assembly, and low-order 3D nodal diffusion approximations to obtain neutron flux distribution throughout the reactor. Then, the detailed fuel pin powers (required for core design and safety analysis) are determined by post-processing the whole core neutron diffusion solution and utilizing pre-computed shape functions, to reconstruct the detailed intra-assembly flux and power distribution. For several decades, these methods - which provide reasonable accuracy and require limited computing resources - have been the workhorse of the nuclear industry. The use of more accurate full-core pin-resolved neutron transport methods to model the reactor requires significant additional computational resources $[1,2,3]$, which are made available through the use of leadership-class computing facilities such as the U.S. Department of Energy INSITE program, which are capable of supporting applications using more than 100,000 compute cores and occasionally hardware accelerators such as GPUs. However, in order to deploy whole core transport methods to the nuclear industry, a methodology is needed that can run on industry-class computing clusters, which are typically between 500 and 5,000 compute cores.

During the last few years, the Method of Characteristics (MOC) has become a nuclear industry standard for solving the transport equation for fuel assembly-sized problems $[4,5]$, to generate the few-group homogenized cross sections for whole-core nodal diffusion methods [6]. Because of the computational appeal of MOC and the familiarity of the industry with this method, 
several researchers investigated the extension of MOC to larger 3D reactor problems $[7,8,2]$. However, it became evident that even with leadership-class computing platforms, the MOC method is too costly for these problems. A group of Korean researchers then investigated " $2 \mathrm{D} / 1 \mathrm{D}$ " methods, which utilize MOC in the 2D radial ( $x$ and $y$ ) directions and a lower-order transport solution in the $1 \mathrm{D}$ axial $(z)$ direction $[9,10]$. This approach was motivated by the fact that most of the material heterogeneity in Light Water Reactors (LWRs) occurs in the radial directions, whereas the axial material heterogeneity is comparatively minimal.

The first 2D/1D method was introduced as the "2D/1D Fusion" method in the CRX code [11], which utilized a 2D MOC solution radially with a discrete-ordinates solution axially. Specifically, the radial 2D MOC method was discretized on a "fine" radial grid (in which each pin cell is divided into 50-100 "fine" spatial cells or "flat source regions"), while the axial solution was discretized on a "coarse" radial cell (consisting typically of one pin cell). The second major implementation of the 2D/1D method was in the DeCART code, developed at the Korean Atomic Energy Research Institute (KAERI) [9]. This method differed from the 2D/1D fusion method in that the axial solver was based on the diffusion approximation (and later, on $\mathrm{SP}_{N}$ ) [10]. A more recent implementation of the $2 \mathrm{D} / 1 \mathrm{D}$ method in DeCART has been the nTRACER code [12]. In the KAERI codes, the 2D MOC methods are also discretized on a fine radial grid, and the axial methods on a coarse radial grid. During the past few years, the KAERI 2D/1D methods have achieved success for practical reactor applications [13].

However, significant limitations in the numerical stability and accuracy were observed in DeCART, particularly when refining the axial mesh. Nonethe-less, the general concept of a 2D/1D method for whole-core reactor methods research provided a useful starting point when the U.S. Department of Energy (DOE) initiated the Nuclear Reactor Simulation Hub, CASL, in 2010. The first step in this development was to derive the 2D/1D equations directly from the 3D transport equation and to formalize the sequence of approximations used in the derivation. The result of this work was a numerically robust 2D/1D method that provided the foundation for the MPACT computer code - which has become the whole-core deterministic neutron transport solver for the CASL core simulator VERA-CS. The purpose of this paper is to describe the principal features of the $2 \mathrm{D} / 1 \mathrm{D}$ method in MPACT.

MPACT is a 3D whole-core transport code based on the 2D/1D method. It provides pin-resolved flux and power distributions, which are important 
for the "challenge problems" in CASL. This fine spatial resolution is achieved by obtaining transport solutions for heterogeneous reactor problems in which the detailed geometrical configuration of fuel components, such as the pellet and cladding, is explicitly retained. The cross-section data needed for the calculation is obtained directly from a multigroup microscopic cross section library, similar to libraries used in lattice physics codes that generate the few-group cross sections for the full-core nodal simulators. Because MPACT involves neither a priori homogenization nor group condensation for the core spatial solution, it represents a significant advance in the fidelity and accuracy of the full-core flux solution, without compromising the stability and robustness required for industry applications.

To provide a sense of the magnitude of the 3D problems solved by MPACT and other 2D/1D codes, a typical reactor core contains:

- $N_{f a} \approx 200$ fuel assemblies,

- $N_{p c / f a} \approx 17 \times 17=289$ pin cells per fuel assembly.

Furthermore, a typical reactor core is discretized using:

- $N_{f c / p c} \approx 50$ fine $2 \mathrm{D}$ spatial cells ("flat source regions") per pin cell,

- $N_{f c}=N_{f c / p c} \cdot N_{p c / f a} \cdot N_{f a} \approx 2.4 \times 10^{6}$ fine $2 \mathrm{D}$ spatial cells,

- $N_{c c}=N_{p c / f a} \cdot N_{f a} \approx 6 \times 10^{4}$ coarse $2 \mathrm{D}$ spatial cells,

- $N_{a x} \approx 400$ axial cells,

- $N_{e g} \approx 50$ energy groups,

- $N_{a q} \approx 120$ directions in the angular quadrature set,

- $N_{t o t}=N_{f c} \cdot N_{a x} \cdot N_{e g} \cdot N_{a q} \approx 10^{13} \approx$ total number of primary unknowns.

However, due to the details of the Method of Characteristics, which effectively imposes a finer radial spatial grid on the problem than the one indicated above, the actual total number of numerical unknowns is often approximately two orders of magnitude greater. For time-dependent simulations (reactor safety or depletion), the time-variable $t$ must also be included, in addition to space, energy, and direction-of-flight. The size of these problems is the principal reason why, until recently, it was not realistically possible to simulate them using standard 3D neutron transport methods.

The following sections of this paper describe a theoretical basis for the 2D/1D method. (Some of the basic theoretical issues underpinning the 2D/1D method are discussed in greater detail in $[14,15]$.) Then some of the 
implementation details are discussed, including the code design and treatments of the transverse leakage. Finally, the 2D/1D method in MPACT is used to simulate problems for realistic LWR geometries, and results are compared to continuous-energy Monte Carlo simulations for the initial critical condition of an operating power plant.

\section{Theory}

In the $2 \mathrm{D} / 1 \mathrm{D}$ capability in MPACT, the 3D domain is decomposed into $2 \mathrm{D}$ planes in which the transport equation is solved. The planes are coupled together through an isotropic leakage source, and the axial variation in the flux is modeled using a lower-order transport approximation discretized by either a nodal or finite difference method. The iterative convergence of the $3 \mathrm{D}$ solution is accelerated using a Coarse Mesh Finite Difference (CMFD) solver.

\subsection{Radial Solver Governing Equations}

The 2D/1D methodology starts with the multigroup Boltzmann transport equation:

$$
\begin{aligned}
\boldsymbol{\Omega} \cdot \boldsymbol{\nabla} \psi_{g}(\boldsymbol{r}, \boldsymbol{\Omega})+ & \Sigma_{t, g}(\boldsymbol{r}) \psi_{g}(\boldsymbol{r}, \boldsymbol{\Omega}) \\
= & \frac{\chi_{g}(\boldsymbol{r})}{4 \pi k_{\mathrm{eff}}} \sum_{g^{\prime}=1}^{G} \nu \Sigma_{f, g^{\prime}}(\boldsymbol{r}) \int_{4 \pi} \psi_{g^{\prime}}\left(\boldsymbol{r}, \boldsymbol{\Omega}^{\prime}\right) d \Omega^{\prime} \\
& \quad+\sum_{g^{\prime}=1}^{G} \int_{4 \pi} \Sigma_{s, g^{\prime} \rightarrow g}\left(\boldsymbol{r}, \boldsymbol{\Omega}^{\prime} \cdot \boldsymbol{\Omega}\right) \psi_{g^{\prime}}\left(\boldsymbol{r}, \boldsymbol{\Omega}^{\prime}\right) d \Omega^{\prime} .
\end{aligned}
$$

For this discussion, the transport-corrected, isotropic scattering approximation $[16,17]$ is made for simplification, but treating higher order approximations to the scattering is a straightforward extension. Thus, we assume:

$$
\Sigma_{s, g^{\prime} \rightarrow g}\left(\boldsymbol{r}, \boldsymbol{\Omega}^{\prime} \cdot \boldsymbol{\Omega}\right) \approx \frac{\Sigma_{s, t r, 0, g^{\prime} \rightarrow g}(\boldsymbol{r})}{4 \pi} .
$$

Now the multigroup transport equation can be written as:

$$
\boldsymbol{\Omega} \cdot \boldsymbol{\nabla} \psi_{g}(\boldsymbol{r}, \boldsymbol{\Omega})+\Sigma_{t r, g}(\boldsymbol{r}) \psi_{g}(\boldsymbol{r}, \boldsymbol{\Omega})=q_{g}(\boldsymbol{r}),
$$


where

$$
\begin{gathered}
q_{g}(\boldsymbol{r})=\frac{\chi_{g}(\boldsymbol{r})}{4 \pi k_{\mathrm{eff}}} \sum_{g^{\prime}=1}^{G} \nu \Sigma_{f, g^{\prime}}(\boldsymbol{r}) \phi_{g^{\prime}}(\boldsymbol{r})+\frac{1}{4 \pi} \sum_{g^{\prime}=1}^{G} \Sigma_{s, t r, 0, g^{\prime} \rightarrow g}(\boldsymbol{r}) \phi_{g^{\prime}}(\boldsymbol{r}), \\
\phi_{g}(\boldsymbol{r})=\int_{4 \pi} \psi_{g^{\prime}}\left(\boldsymbol{r}, \boldsymbol{\Omega}^{\prime}\right) d \Omega^{\prime} .
\end{gathered}
$$

By averaging the 3D-Boltzmann transport equation axially (as in Eq. 6), Eq. 7 is obtained:

$$
\begin{gathered}
\psi_{g}^{k}(x, y, \boldsymbol{\Omega})=\frac{1}{h_{z}^{k}} \int_{z_{B^{k}}}^{z_{T^{k}}} \psi_{g}(\boldsymbol{r}, \boldsymbol{\Omega}) \\
\left(\Omega_{x} \frac{\partial}{\partial x}+\Omega_{y} \frac{\partial}{\partial y}\right) \psi_{g}^{k}(x, y, \boldsymbol{\Omega})+\sum_{t, g}^{k} \psi_{g}^{k}(x, y, \boldsymbol{\Omega})=\tilde{q}_{g}^{k}(x, y, \boldsymbol{\Omega}),
\end{gathered}
$$

where $\tilde{q}$ consists of the scattering and fission sources in $q$ (see Eq. 4). The axial component of the streaming operator becomes the axial transverse leakage term:

$$
\begin{gathered}
\tilde{q}_{g}^{k}(x, y, \boldsymbol{\Omega})=q_{g}^{k}(x, y, \boldsymbol{\Omega})+T L_{g}^{k}(x, y, \boldsymbol{\Omega}), \\
T L_{g}^{k}(x, y, \boldsymbol{\Omega})=\frac{\Omega_{z}}{h_{z}^{k}}\left(\psi_{B^{k}, g}(x, y, \boldsymbol{\Omega})-\psi_{T^{k}, g}(x, y, \boldsymbol{\Omega})\right) .
\end{gathered}
$$

A simple approximation is to assume that the axial transverse leakage is isotropic. This leakage is obtained by averaging Eq. 7c over $\Omega$ :

$$
T L_{g}^{k}(x, y) \approx \frac{\left(J_{B^{k}, g}(x, y)-J_{T^{k}, g}(x, y)\right)}{4 \pi h_{z}^{k}} .
$$

This has several computational advantages that are discussed in the following sections.

Another approximation is to assume that the transverse leakage is spatially flat (see Eq. 9) across the entire pin. By averaging Eq. 7c radially, the equation for the flat approximation can be found using both the explicit angular form (Eq. 9a) and the $0^{\text {th }}$ moment integral (Eq. 9b):

$$
\begin{gathered}
T L_{g}^{k, X Y}(\boldsymbol{\Omega})=\frac{\Omega_{z}}{h_{z}^{k}}\left(\psi_{B^{k}, g}^{X Y}(\boldsymbol{\Omega})-\psi_{T^{k}, g}^{X Y}(\boldsymbol{\Omega})\right), \\
T L_{g, 0}^{k, X Y}=\int_{4 \pi} T L_{g}^{k, X Y}(\boldsymbol{\Omega}) d \Omega=\frac{\left(J_{B^{k}, g}^{X Y}-J_{T^{k}, g}^{X Y}\right)}{4 \pi h_{z}^{k}} .
\end{gathered}
$$




\subsection{Axial Solver Governing Equations}

The axial equations can be derived in a similar manner by averaging the 3D transport equation radially over both $x$ and $y$, as in Eq. 10, yielding Eq. 11. In MPACT, the axial solver operates on the pin-wise basis, so the bounds of $x$ and $y$ correspond to the coarse radial grid. This leaves only a dependence on $z$, but with a radial transverse leakage in the source, as shown in Eq. 11d:

$$
\begin{gathered}
\psi_{g}^{X Y}(z, \boldsymbol{\Omega})=\frac{1}{A_{x y}} \int_{x_{L}}^{x_{R}} \int_{y_{L}}^{y_{R}} \psi_{g}(\boldsymbol{r}, \boldsymbol{\Omega}) d y d x \\
\Omega_{z} \frac{\partial}{\partial z} \psi_{g}^{X Y}(z, \boldsymbol{\Omega})+\Sigma_{t, g} \psi_{g}^{X Y}(z, \boldsymbol{\Omega})=\tilde{q}_{g}^{X Y}(z, \boldsymbol{\Omega}) \\
\tilde{q}_{g}^{X Y}(z, \boldsymbol{\Omega})=\bar{q}_{g}^{X Y}(z, \boldsymbol{\Omega})+T L_{g}^{X Y}(z, \boldsymbol{\Omega}) \\
\bar{q}_{g}^{X Y}(z, \boldsymbol{\Omega})=\frac{\chi_{g}}{4 \pi k_{\mathrm{eff}}} \sum_{g^{\prime}=1}^{N_{g}} \nu \Sigma_{f, g^{\prime}} \phi_{g^{\prime}}^{X Y}(z)+\frac{1}{4 \pi} \sum_{g^{\prime}=1}^{N_{g}} \Sigma_{s, g^{\prime} \rightarrow g} \phi_{g}^{X Y}(z) \\
T L_{g}^{X Y}(z, \boldsymbol{\Omega})=-\frac{1}{A_{x y}}\left(\begin{array}{c}
\Omega_{x} \int_{y_{L}}^{y_{R}}\left(\psi_{g}\left(x_{R}, y, z, \boldsymbol{\Omega}\right)-\psi_{g}\left(x_{L}, y, z, \boldsymbol{\Omega}\right)\right) d y \\
+\Omega_{y} \int_{x_{L}}^{x_{R}}\left(\psi_{g}\left(x, y_{R}, z, \boldsymbol{\Omega}\right)-\psi_{g}\left(x, y_{L}, z, \boldsymbol{\Omega}\right) d y\right.
\end{array}\right)
\end{gathered}
$$

The axial solvers covered in this work use an isotropic radial transverse leakage, in which case the transverse leakage has no angular dependence and can be constructed using the net currents at the right and left boundaries of each coarse radial cell, as in Eq. 12:

$$
T L_{g}^{X Y}(z, \Omega)=\frac{1}{4 \pi h_{x}}\left(J_{L, x, g}(z)-J_{R, x, g}(z)\right)+\frac{1}{4 \pi h_{y}}\left(J_{L, y, g}(z)-J_{R, y, g}(z)\right) .
$$

\subsection{D $M O C$}

The 2D MOC transport sweeper in MPACT has been previously reported $[4,18,19]$, provides the foundation of the 2D/1D sweeper. Although any $2 \mathrm{D}$ solver could potentially be used in the $2 \mathrm{D} / 1 \mathrm{D}$ formulation, the basis of MPACT is the 2D MOC sweeper. The assumption in the 2D/1D method is that the axial transverse leakage source is isotropic, as was discussed in

Section 2.1. Although some angular information is available concerning the axial leakage, the isotropic assumption provides significant advantages in 
terms of speed and memory for the 2D MOC solution. Ongoing research is investigating computationally efficient methods to include the angular shape of the axial leakage in the $2 \mathrm{D} / 1 \mathrm{D}$ solution.

The characteristic form of the transport equation can be obtained by casting a characteristic direction $\boldsymbol{r}$ and rewriting Eq. 3.

$$
\begin{gathered}
\boldsymbol{r}=\boldsymbol{r}_{\mathbf{0}}+s \boldsymbol{\Omega}=\left\{\begin{array}{l}
x=x_{0}+s \Omega_{x}, \\
y=y_{0}+s \Omega_{y}, \\
z=z_{0}+s \Omega_{z},
\end{array}\right. \\
\frac{d \psi_{g}\left(\boldsymbol{r}_{\mathbf{0}}+s \boldsymbol{\Omega}, \boldsymbol{\Omega}\right)}{d s}+\Sigma_{t, g}\left(\boldsymbol{r}_{\mathbf{0}}+s \boldsymbol{\Omega}\right) \psi_{g}\left(\boldsymbol{r}_{\mathbf{0}}+s \boldsymbol{\Omega}, \boldsymbol{\Omega}\right)=q_{g}\left(\boldsymbol{r}_{\mathbf{0}}+s \boldsymbol{\Omega}, \boldsymbol{\Omega}\right) .
\end{gathered}
$$

This ordinary differential equation is then solved directly along a characteristic ray through a region with a constant cross section and constant source:

$$
\psi_{g}(s, \boldsymbol{\Omega})=\psi_{g}\left(s_{0}, \boldsymbol{\Omega}\right) \exp \left(\Sigma_{t, g} s\right)+\frac{q_{g}}{\Sigma_{t, g}}\left(1-\exp \left(\Sigma_{t, g} s\right)\right) .
$$

With this equation, the MOC sweeping scheme can be devised. Individual rays are traced across the geometry to generate segment information on the spatial mesh. The spatial discretizations that are available include any combination of unstructured Cartesian and cylindrical/azimuthal mesh. The two quantities of interest for the MOC sweep are the outgoing angular flux and the average angular flux across a given segment, $l$, in a fine mesh region, $r$, both of which can be obtained from Eq. 14:

$$
\begin{gathered}
\psi_{g, l, r}^{\text {out }}=\psi_{g, l, r}^{\text {in }} \exp \left(\Sigma_{t, g, r} s_{l, r}\right)+\frac{q_{g, r}}{\sum_{t, g, r}}\left(1-\exp \left(\Sigma_{t, g, r} s_{l, r}\right)\right), \\
\bar{\psi}_{g, l, r}=\frac{\psi_{g, l, r}^{\text {in }}-\psi_{g, l, r}^{\text {out }}}{\sum_{t, g, r} s_{l, r}}+\frac{q_{g, r}}{\sum_{t, g, r}} .
\end{gathered}
$$

Up to this point, there has been no discussion about how the rays are selected and laid across the geometry. The direction of the rays are chosen based on a quadrature set. For most of the solvers in MPACT, the azimuthal and polar angles are selected independently and the product of the two are used. In the azimuthal direction, equally spaced angles are chosen with equal weights, but in order to reduce the memory footprint of the MOC solver, the 
angles are adjusted to maintain a repeatable set of rays across the whole geometry.

The polar angles are chosen based on whether the geometry is $2 \mathrm{D}$ or $3 \mathrm{D}$. For 2D geometries, the Tabuchi-Yamamoto (T-Y) quadrature set is used [20]. This quadrature set was developed to optimize the solution of the 2D transport equation by selecting angles and weights that minimize the error of the solution to the 2D collision probability method. For 3D problems, optimizing the quadrature set in this manner does not make sense because the T-Y quadrature set does not preserve even the low-order limits of the 3D transport equation, including the asymptotic diffusion limit. Hence, for 3D cases, the polar angles and weights are chosen using a 1D Gauss-Legendre quadrature set.

MPACT allows the user to spatially decompose the geometry using Message Passing Interface (MPI) by treating each subdomain as an independent fixed-source problem during each sweep. The incoming angular flux on each subdomain is the outgoing angular flux from the previous sweep of the neighboring subdomain. In addition to spatial decomposition, the angular variable can be decomposed using MPI. The total number of MPI processes must be

equal to the product of the number of spatial domains and the number of angular domains. On each subdomain, finer grain parallelism is achieved by threading the characteristic rays using OpenMP.

\subsection{D Axial Solver}

The axial direction is solved using a low-order approximation to the transport equation. There are two primary axial solvers in MPACT: the nodal expansion method (NEM) solver, which uses a two-node kernel formulation based on the diffusion approximation, and the simplified $P_{n}\left(S P_{n}\right)$ solver, which assumes that the angular flux is represented by a higher order Legendre expansion angularly and wraps a one-node NEM kernel to handle the spatial distribution.

\subsubsection{Diffusion with NEM}

In the nodal expansion method, the source is expanded using quadratic Legendre polynomials and the flux is expanded using quartic polynomials $[21]$ :

$$
Q_{g}(\xi)=\sum_{i=0}^{2} q_{g, i} P_{i}(\xi)
$$




$$
\phi_{g}(\xi)=\sum_{i=0}^{4} a_{g, i} P_{i}(\xi),
$$

where $\xi$ is the normalized axial variable from -1 to 1 and $P_{i}(\xi)$ is the $i^{\text {th }}$ Legendre polynomial. One major difference between the formulation used here and the traditional NEM method proposed by Finnemann is that in this work, the range of the normalized spatial variable is from -1 to 1 instead of the traditional 0 to 1 range. Using -1 to 1 is advantageous because of the orthogonal properties of Legendre Polynomials.

The coefficients $\left(a_{i}\right)$ are determined from the $0^{\text {th }}$ through $2^{\text {nd }}$ moment balance equations (Eq. 18a) in conjunction with flux and current continuity enforcement (Eq. 18c and 18d). These flux coefficients are then used to construct the source coefficients $\left(q_{i}\right)$ for the next iteration.

$$
\begin{gathered}
\int_{-1}^{1} P_{n}(\xi)\left(-\Sigma_{D, g} \frac{d^{2}}{d \xi^{2}} \phi(\xi)+\Sigma_{r, g} \phi(\xi)-Q_{g}(\xi)\right) d \xi=0, \\
\Sigma_{D}=\frac{4 D}{h^{2}} \\
\phi_{1, g}(1)=\phi_{2, g}(-1), \\
J_{1, g}(1)=J_{2, g}(-1) .
\end{gathered}
$$

Lastly, the additional constraint needed to solve the two-node problem is that the average flux in both of the adjacent nodes are preserved. Preserving the average flux of each node serves to stabilize the local iteration and helps prevent the two-node solutions from becoming out of phase with the global solution. The boundary nodes are solved in a slightly different manner to account for the boundary conditions.

Because the radial MOC solvers account for a large majority of the overall run time, the dominant parallel decomposition in the $2 \mathrm{D} / 1 \mathrm{D}$ problem is by axial planes, where the axial solution is highly decomposed. In the axial iteration scheme, each process is responsible for calculating the two-node problems for the cells it owns and the top neighboring cell. With this strategy, the shapes of the source, cross sections, and the average flux are passed via MPI communication to the bottom neighboring processor. This will solve the two-node problem, and then the flux shape and interface current are passed back to the top neighboring processor. In the case of the bottom boundary of the core, the processor that owns the bottom node will also solve for that interface. 


\subsection{2. $S P_{N}$ with $N E M$}

In 1D, the simplified $\mathrm{P}_{N}$ and full $\mathrm{P}_{N}$ equations are identical. However, the term simplified $\mathrm{P}_{N}$ is commonly used to emphasize that these methods are not as burdensome as the full, multidimensional $\mathrm{P}_{N}$ equations can be. With this method, the angular flux, which has been azimuthally integrated, is assumed to have a Legendre expansion in $\mu$ [22]:

$$
\psi_{g}(z, \mu)=\sum_{m=0}^{N_{m o m}} \frac{2 m+1}{2} \psi_{m, g}(z) P_{m}(\mu) .
$$

By substituting Eq. 19 into the transport equation, multiplying by $P_{n}(\mu)$, and integrating over $\mu$, Eq. 20a can be obtained for each moment,

$$
\begin{aligned}
\frac{d}{d z}\left[\frac{n}{2 n+1} \psi_{n-1, g}(z)+\frac{n+1}{2 n+1} \psi_{n+1, g}(z)\right] & +\Sigma_{t, g}(z) \psi_{n, g}(z) \\
= & \Sigma_{s n, g \rightarrow g} \psi_{n, g}(z)+Q_{g}(z) \delta_{n, 0}
\end{aligned}
$$

and with the corresponding boundary conditions (Eqs. 20b and 20c) for the bottom and top interfaces, respectively:

$$
\begin{aligned}
& \sum_{m=0}^{N_{m o m}} \frac{2 m+1}{2}\left(\int_{-1}^{0} \mu P_{n}(\mu) P_{m}(\mu) d \mu\right) \psi_{m, g}(0)=\int_{0}^{1} \mu P_{n}(\mu) \psi_{g}^{b}(\mu) d \mu, \\
& \sum_{m=0}^{N_{m o m}} \frac{2 m+1}{2}\left(\int_{-1}^{0} \mu P_{n}(\mu) P_{m}(\mu) d \mu\right) \psi_{m, g}(Z)=\int_{-1}^{0} \mu P_{n}(\mu) \psi_{g}^{b}(\mu) d \mu .
\end{aligned}
$$

In MPACT, $\mathrm{SP}_{3}$ and $\mathrm{SP}_{5}$ can be used, which can be formulated to wrap the one-node NEM kernel [23]. With this setup, $\mathrm{SP}_{N}$ handles the angular distribution and NEM handles the spatial distribution. The details of the derivation will be omitted here, but once the equations for the $0^{\text {th }}$ through $3^{\text {rd }}$ moments have been found, the first and third can be solved and substituted into the $0^{\text {th }}$ and $2^{\text {nd }}$ moment equations. Additionally, the boundary condition equations need to be slightly rearranged to yield the partial surface flux moment, as is anticipated for the one-node NEM kernel. In the current implementation, the higher order scattering cross sections are neglected, but will be incorporated in future work. This solver generates updated partial currents from the $0^{\text {th }}$ moment equation ( $n=0$ in Eq. $20 \mathrm{~b}$ and $20 \mathrm{c}$ ), which are then used to construct the axial transport leakage for the radial solver and the current correction coupling coefficients for CMFD. 


\subsubsection{Radial Transverse Leakage Interpolation}

It is important to note that in Eq. 12 the currents that come from the radial sweeper have no axial dependence. However, since most of the axial sweepers use some form of axial Legendre expansion of the spatial shape, they have the ability to incorporate higher-order transverse leakage components into the source construction using the transverse leakages from the neighboring planes.

$$
\begin{gathered}
T L_{g}^{X Y}(z)=\sum_{i=0}^{2} T L_{g, i}^{X Y} P_{i}(\xi), \\
G=2\left(h_{C}+h_{B}\right)\left(h_{C}+h_{T}\right)\left(h_{B}+h_{C}+h_{T}\right), \\
T L_{g, 0}^{X Y}=T L_{C, g}^{X Y}, \\
T L_{g, 1}^{X Y}=G^{-1} h_{C}\left[\left(T L_{T, g}^{X Y}-T L_{C, g}^{X Y}\right)\left(h_{C}+2 h_{B}\right)\left(h_{C}+h_{B}\right)\right. \\
\left.-\left(T L_{B, g}^{X Y}-T L_{C, g}^{X Y}\right)\left(h_{C}+2 h_{T}\right)\left(h_{C}+h_{T}\right)\right], \\
T L_{g, 2}^{X Y}=G^{-1}\left(h^{c}\right)^{2}\left[\left(T L_{g}^{X Y, t}-T L_{g}^{X Y, c}\right)\left(h^{c}+h^{b}\right)\right. \\
\left.\left.+\left(T L_{g}^{X Y, b}-T L_{g}^{X Y, c}\right)\right)\left(h^{c}+h^{t}\right)\right],
\end{gathered}
$$

where $h_{C}, h_{B}$, and $h_{T}$ correspond to the axial node height of the center, bottom, and top neighboring planes, respectively, and $g$ denotes the group index. Eq. 21a describes the objective to determine the axial shape of the axial leakage as a series of Legendre Polynomial. Using the neighboring information from the top and bottom nodes, Eq. 21b provides the geometric information needed for the denominator of all of the coefficients. Eq. 21c, 21d, and 21e represent the flat, linear, and quadratic coefficients for the Legendre expansion in Eq. 21a by using the neighboring information.

In the above equations, the maximum expansion is quadratic, as this is the highest that can be obtained with only the neighboring plane information. It is feasible to use cubic or even quartic expansions, but this would require data from second or third nearest neighboring planes and more data-passing between spatial decomposition processors. 


\subsection{Coarse-Mesh Finite-Difference (CMFD) Acceleration}

The CMFD acceleration scheme $[24,25]$ not only accelerates the convergence of the entire solution but also provides stability to the $2 \mathrm{D} / 1 \mathrm{D}$ solver $[14,15]$. Since the radial and axial equations are solved alternately, the tendency is for data propagation in the axial direction to become very slow as the planes become thin. CMFD helps couple all of the planes together by solving a global diffusion-like system of equations. The elliptic nature of the diffusion system tightly couples the interior of the core to the boundary.

The CMFD acceleration is implemented to enhance the spatial and energy convergence. CMFD is a nonlinear iteration scheme that accelerates the convergence of the eigenfunction and eigenvalue. CMFD is based on the $0^{\text {th }}$ angular moment of the transport equation on a coarse mesh:

$$
\begin{aligned}
\boldsymbol{\nabla} \cdot \boldsymbol{J}_{\boldsymbol{g}}(\boldsymbol{r})+ & \Sigma_{t, g}(\boldsymbol{r}) \phi(\boldsymbol{r}) \\
& =\sum_{g^{\prime}=1}^{G} \Sigma_{s, 0, g^{\prime} \rightarrow g}(\boldsymbol{r}) \phi_{g^{\prime}}(\boldsymbol{r})+\frac{\chi_{g}(\boldsymbol{r})}{k_{\mathrm{eff}}} \sum_{g^{\prime}=1}^{G} \nu \Sigma_{f, g^{\prime}}(\boldsymbol{r}) \phi_{g^{\prime}}(\boldsymbol{r}) .
\end{aligned}
$$

This equation exactly preserves neutron balance in every coarse mesh region, but it cannot be solved by itself because there is not a defined relationship between the scalar flux and the neutron current. This constraint is obtained by starting with the finite-difference form of the diffusion equation through a diffusion coefficient, $\widetilde{D}_{g, s}$, times the difference between the neighboring fluxes. The $\widetilde{D}_{g, s}$ term used here is a harmonic mean of the diffusion coefficients on the positive and negative side of surface $s$ divided by the distance between the two mesh centroids, $\delta$ :

$$
\widetilde{D}_{g, s}=\frac{2 D_{g}^{+} D_{g}^{-}}{\delta\left(D_{g}^{+}+D_{g}^{-}\right)} .
$$

A second term is added that incorporates the sum of the two neighboring nodes times an undefined coefficient, $\widehat{D_{s}}$ :

$$
J_{g, s}=-\widetilde{D}_{g, s}\left(\bar{\phi}_{g}^{+}-\bar{\phi}_{g}^{-}\right)+\widehat{D}_{g, s}\left(\bar{\phi}_{g}^{+}+\bar{\phi}_{g}^{-}\right)
$$

where $\bar{\phi}_{g}^{+}$and $\bar{\phi}_{g}^{-}$represent the scalar flux on the positive and negative sides of surface $s$.

The $\widehat{D}_{g, s}$ coefficient is obtained by enforcing the current from the solution of the transport equation obtained by $\mathrm{MOC}$ in the radial direction and 
the nodal solution in the axial on the coarse mesh cells. The solution is homogenized across each pin cell and the currents are calculated on each pin boundary. With this data, the $\widehat{D}_{g, s}$ term can be defined:

$$
\widehat{D}_{g, s}=\frac{J_{g, s}^{\mathrm{MOC}}+\widetilde{D}_{g, s}\left(\bar{\phi}_{g}^{+}-\bar{\phi}_{g}^{-}\right)}{\left(\bar{\phi}_{g}^{+}+\bar{\phi}_{g}^{-}\right)} .
$$

The cross-sections for each coarse mesh region region are homogenized using scalar flux-volume weighting to preserve the reaction rates. Figure 1 shows the relationship between the fine MOC mesh (left) and the coarse CMFD mesh (right).

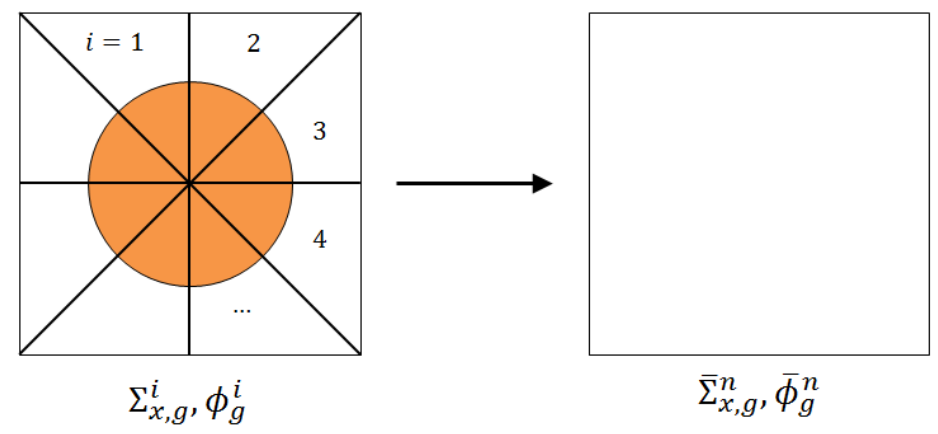

Figure 1: Coarse Mesh Homogenization

The cross-sections and scalar flux are homogenized as follows:

$$
\begin{gathered}
\bar{\Sigma}_{C, x, g}=\frac{\sum_{i \in C} \sum_{i, x, g} \phi_{i, g} V_{i}}{\sum_{i \in C} \phi_{i, g} V_{i}}, \\
\bar{\phi}_{C, g}=\frac{\sum_{i \in C} \phi_{i, g} V_{i}}{\sum_{i \in C} V_{i}},
\end{gathered}
$$

where $C$ is the coarse cell index, $i$ is the fine cell index, and $x$ is an arbitrary reaction type.

With the definitions of $J_{g, s}, \widehat{D}_{g, s}, \bar{\Sigma}_{C, x, g}$, and $\bar{\phi}_{C, x, g}$, a system of equations is then constructed and solved. Once this system of equations is solved, the MOC flat source region fluxes are updated and the 2D/1D sweep is repeated. This process continues until the change in eigenvalue and two-norm of the fission source meet the specified convergence criteria. 


\section{Implementation}

Now that all of the components of the 2D/1D sweeper have been presented, the iteration strategy will be explained, as well as several of the special considerations that need to be made. First, a brief discussion of a few features of the architecture and 2D/1D iteration scheme is given. Then the issue of the negative sources caused by large, negative transverse leakages is addressed. Next, issues that arise from the spatial distribution of the axial transverse leakage as applied to the radial solver are discussed. Finally, the application of stability analysis to MPACT is described.

\subsection{Architecture}

The architecture of MPACT is documented in detail in [26], but an overview of a few key components is provided here in order to understand how the different components fit together. MPACT's design is based on object-oriented programming, including the solver taxonomy, which includes solvers ranging from simulating multiple states and handling the long-term time-dependence of the isotopic inventory, to eigenvalue solvers, to solvers that handle group sweeping for fixed-source problems, and finally to a onegroup transport sweep. All of the different components we are discussing MOC, 1D NEM, 1D $\mathrm{SP}_{N}$, and 2D/1D - are all transport sweepers that use the same eigenvalue and fixed-source solver interfaces.

The $2 \mathrm{D} / 1 \mathrm{D}$ transport sweeper is unique in that it contains references to several other transport sweepers. It contains a vector of $2 \mathrm{D}$ transport sweepers that each represent a plane, and an axial transport sweeper that couples the planes together. Additionally, it is in charge of iterating across all the 2D planes, iterating across all of the 1D axial solves, and providing transverse leakages between the radial and axial solvers.

Accelerator objects can also be enabled for the eigenvalue, fixed source, or transport sweep solvers. In this case, the CMFD acceleration algorithm is implemented to accelerate the eigenvalue problem directly.

In order to take advantage of larger machines, MPACT uses MPI to decompose the 3D domain into both the spatial and angular dimensions. It also uses OpenMP to provide threading capability for the MOC rays. The spatial domain for $2 \mathrm{D} / 1 \mathrm{D}$ problems is preferentially decomposed in the axial direction first to enhance parallel performance of the $2 \mathrm{D}$ transport solves on each plane. MPACT also has the ability to decompose each radial plane once all the planes are decomposed. 


\subsection{Iteration Scheme}

The 2D/1D iteration scheme begins with a 3D CMFD solve with the $\widehat{D}$ correction terms set to zero and the cross-sections homogenized using a uniform flux distribution. This provides a very preliminary "diffusion" fission source distribution throughout the core. This cell-wise fission source is then used to run the 2D MOC calculation on every plane and transverse leakage source that is derived from the CMFD solution. The 2D MOC solution calculates the net neutron currents on the pin cell mesh. With these radial currents, the axial solve can be performed to calculate the net neutron currents in the axial direction. Then the CMFD is repeated with updated $\widehat{D}$ terms from the radial and axial solves. This process is repeated until all of the convergence criteria are met.

\subsection{Transverse Leakage Splitting}

In both the radial and axial equations, difficulties can occur when the transverse leakage terms cause the total source (scattering plus fission plus transverse leakage) to become negative. One scheme used to avoid this is known as transverse leakage splitting. This technique takes the negative transverse leakage (or some component of it) and subtracts it from the total cross section of the collision operator.

For example, consider the 1D diffusion-based axial equation. If the transverse leakage is split to the left-hand side, the equation becomes:

$$
\begin{aligned}
-D_{g} \frac{\partial^{2} \phi_{g}(z)}{\partial z^{2}} & +\left(\Sigma_{r, g}+\frac{T L_{g}^{X Y}(z)}{\phi_{g}(z)}\right) \phi_{g}(z) \\
& =\frac{\chi_{g}(z)}{k_{e f f}} \sum_{g^{\prime}=1}^{N_{g}} \nu \Sigma_{f, g^{\prime}} \phi_{g^{\prime}}(z)+\sum_{g^{\prime}=1, g^{\prime} \neq g}^{N_{g}} \Sigma_{s, g^{\prime} \rightarrow g} \phi_{g^{\prime}}(z) .
\end{aligned}
$$

A similar approach can be made when splitting the transverse leakage for the MOC solver. We can also consider that the self-scattering source (from group g to group g) can be separated from the rest of the source, and thus the source term in Eq. 7a becomes

$$
\tilde{q}_{g}^{Z}(x, y)=q_{g}^{Z}(x, y)+q_{s s, g}^{Z}(x, y)+T L_{g}^{Z}(x, y) .
$$

Several different approaches to splitting have been explored [27], but the most commonly used one in MPACT places the source (only if negative) into the total cross section by dividing the source by the scalar flux for that region. 
Because the self-scattering source updates during each inner iteration, it is not included in the splitting, but remains on the right-hand side;

$$
\begin{aligned}
& \left(\Omega_{x} \frac{\partial}{\partial x}+\Omega_{y} \frac{\partial}{\partial y}\right) \psi_{g}^{Z}(x, y, \Omega) \\
& \quad+\left(\Sigma_{t, g}+\frac{\tilde{q}_{g}^{Z}(x, y)-q_{s s, g}^{Z}(x, y)}{\phi_{g}^{Z}}\right) \psi_{g}^{Z}(x, y, \Omega)=\tilde{q}_{s s, g}^{Z}(x, y) .
\end{aligned}
$$

This approach of isotropically splitting the source reduces the memory required because only one value is stored for all angles. Since this is only done when the source is negative, it allows the calculation to proceed as usual unless problems arise. In general, the effect on accuracy when splitting isotropic sources is negligible.

\subsection{Axial Transverse Leakage Shape Functions}

Eq. 9 shows how the flat axial transverse leakage distribution is derived by using the surface flux moment quantities on the top and bottom axial surfaces, which span the entire pin. This means that all fine-mesh regions within the pin have the same transverse leakage value applied. While this is a useful simplification, it can also pose stability problems, particularly when placing a negative transverse leakage source into a region with little or no

source (i.e., low-density regions), such as the fuel-clad gap. An approach has been implemented that is referred to as the "limited flat" transverse leakage shape, which checks the total cross section of each region. If the cross section value is below $10^{-3} \mathrm{~cm}^{-1}$, the transverse leakage that would be placed into that region is redistributed to other regions with larger cross sections. This is done by tabulating the area of the regions that do not meet the cross section requirements $\left(A_{\text {reject }}\right)$ and formulating a ratio using the total area of the pin, which is then used to scale the transverse leakage values in the other regions,

$$
T L_{g}^{Z}=T L_{g}^{Z} \frac{A_{\text {pin }}}{A_{\text {pin }}-A_{\text {reject }}} .
$$

As can be seen, the scaling ratio will always be greater than or equal to one, to preserve the total leakage rate.

\subsection{Stability Implementation}

In previous work, it was observed that the iteration scheme could be unstable in scenarios where the axial solver does not perform multiple sweeps 
[14]. To stabilize the scheme, a relaxation factor is calculated for each pin, on each plane, and for each energy group,

$$
\theta_{\text {opt }}=\frac{2}{2-\left(\omega_{1, \max }+\omega_{1, \min }\right)}
$$

where $\omega$ represents the growth factor that comes from the Fourier analysis of the 2D/1D method accelerated by CMFD. These growth factors depend on the self-scattering ratio and the axial optical thickness. In order to make the determination of these growth factors computationally tractable, a fit was performed that gives a conservative bound for the relaxation factor [14]. The minimum value of all of the relaxation factors for all spatial regions is determined for each energy group and used to relax the solution. Figure 2 shows the minimum optimal, energy-dependent relaxation factors, which are a function of the axial optical thickness $\left(\tau_{g}\right)$ of each pin and the scattering ratio $\left(c_{g}\right)$ for a test problem. In this figure, two different sets of relaxation factors are shown, the first for a larger axial mesh of $7.6 \mathrm{~cm}$ and the second for a more refined mesh at $1.0 \mathrm{~cm}$. In this case, transport-corrected scattering was used, so the self-scattering cross section, and resultantly scattering ratio, can be negative. Given what is known about the instabilities that occur because of optically thin regions, it makes sense that the under-relaxation is more severe in fast energy groups, where the optical thickness is lower. As expected, the relaxation factors for the $1 \mathrm{~cm}$ mesh are significantly lower than there for the $7.6 \mathrm{~cm}$ mesh. It is also notable that the theory suggests that some energy groups can be over-relaxed (i.e., $\theta_{g}>1$ ). In the current implementation, the relaxation factors are capped at unity, so no over-relaxation is permitted.

Figure 3 shows the 2-norm residual of the fission source distribution as a function of iteration index for a case where finite-difference is used axially, so there are no axial sweeps and the CMFD solver handles the axial transport. The trend with no relaxation $(\theta=1)$ is shown with the solid line. As can be seen, the residual approaches $10^{-5}$ and then begins to diverge. The dashdotted line shows the trend using a group-independent relaxation based on the minimum of all of the groups. The initial attempts at relaxation that occurred before the Fourier analyses focused on applying a constant relaxation factor to all of the groups. In this case, similar behavior is observed where the flux residual monotonically decreases but takes a significant number of iterations to reach convergence. Lastly, the dashed line shows the residual with the group-dependent, optimal relaxation factor. A significant reduction 


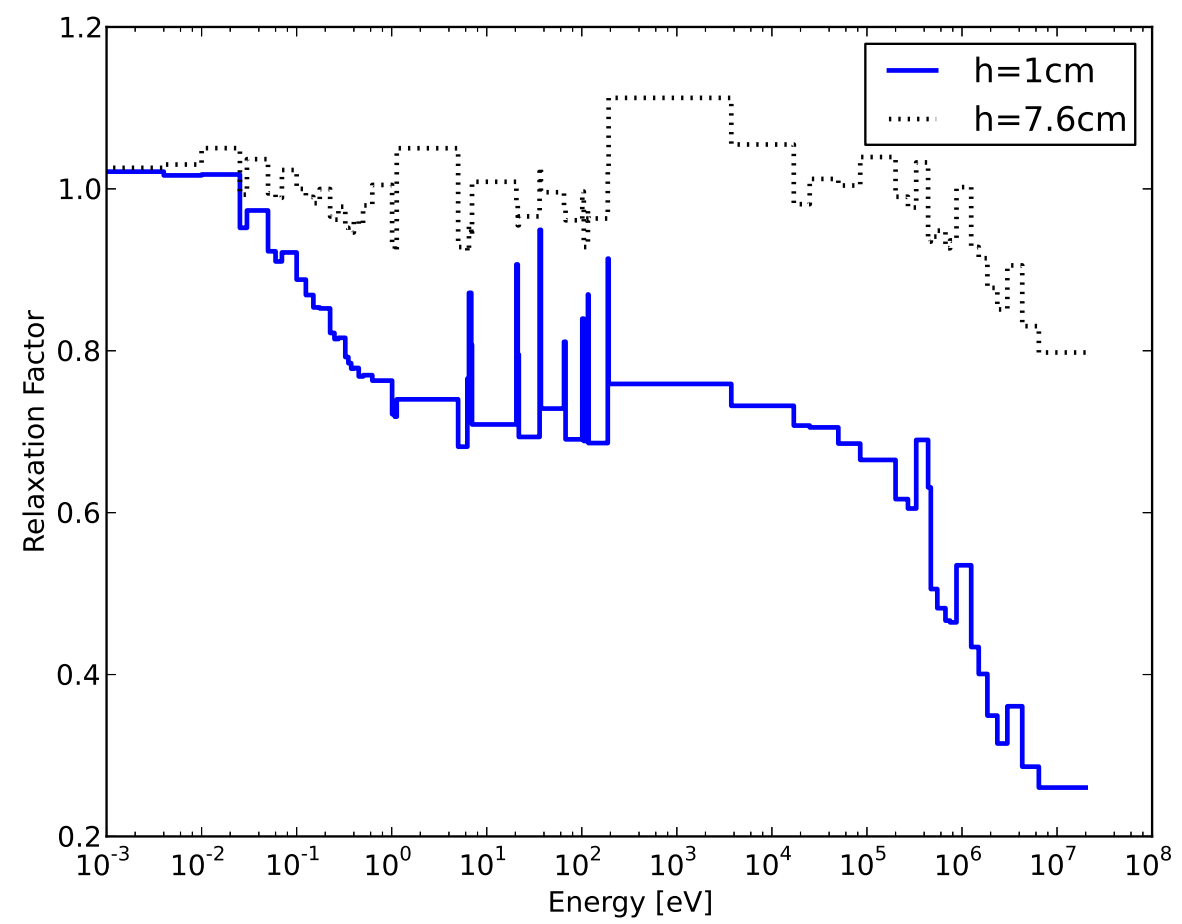

Figure 2: Energy-Dependent, Optimal Relaxation Factors

in the number of iterations is seen compared to using a group-independent relaxation values. The test problem that was used here is not a typical problem, but it illustrates the relaxation techniques well.

The relaxation techniques covered in this section have become an important component of the 2D/1D implementation in MPACT, allowing for explicit axial representation of spacer grids and structural components [28], including Intermediate Flow Mixing (IFM) grids, which can be even smaller than typical spacer grids [29]. The original derivation of the relaxation factors was performed assuming Fick's Law axially and without multiple axial sweeps. To resolve the boundary conditions of the axial $\mathrm{SP}_{N}$ solver, multiple sweeps are performed, which may invalidate the need to relax at all. However, to remain conservatively stable, the same relaxation factors are still in use. Additionally, it is suspected that convergence can be achieved without relaxation in the presence of thin planes if they are isolated and few in num- 


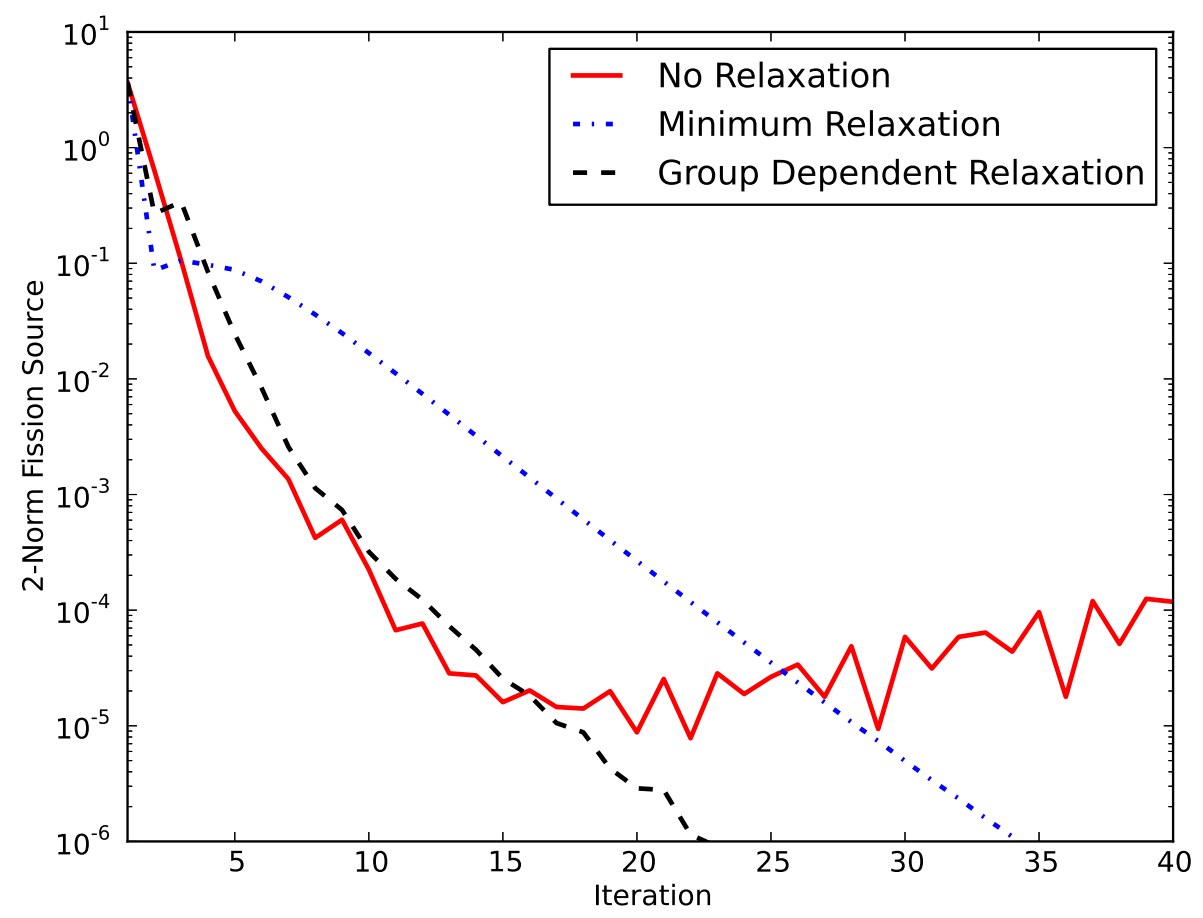

Figure 3: 2-Norm Fission Source Convergence with Various Relaxation Approaches

ber, as would be the case with spacer grids, which are separated by several larger fuel planes.

\section{Application}

The target application for MPACT is full-core reactor geometry with as few simplifications as possible. This includes explicit modeling of the fuel, cladding, and core baffle in the radial direction. In the axial direction, a few approximations are made, but much of the geometry is retained. The spacer grids are placed around the periphery of the pin cells. The mixing vanes are not explicitly modelled, but the grid mass is preserved. The upper and lower nozzles and core plates are homogenized to simplify user input.

The VERA Core Physics Progression problems [28] are used to analyze the accuracy of the solvers in MPACT. The progression problems are set up to first model a 2D pin cell. The pin cell is useful for understanding the 
quality of the the multigroup cross-section library and resonance processing. The second set of problems considers the accuracy of the $2 \mathrm{D}$ solver, including several single-assembly problems, multiassembly problems, and full-core problems. The full-core problem will be considered here. The third and fourth problems start to focus on the accuracy of the 3D solver. The third problem considers a single 3D assembly, and the fourth considers multiple assemblies with a poison rod inserted into the center assembly. The fifth problem considers a full 3D PWR core and models the initial startup of the Watts Bar Nuclear Power Station. The remaining five problems in the progression problems focus on coupling to thermal-hydraulics, which is not a focus of the current work.

In the configurations considered in this work, the cases are compared to a continuous-energy Monte Carlo code, KENO [30], which is part of the SCALE package [31]. KENO provides both eigenvalue and pin power distribution for all cases. Since there are significantly fewer approximations made in Monte Carlo codes, they are usually used as a reference when measured data is not available. For the full-core configuration, measured eigenvalues for $10 \mathrm{critical}$ configurations are also available.

\subsection{D Core}

The first case considered is the $2 \mathrm{D}$ core. This case demonstrates the accuracy of the $2 \mathrm{D}$ MOC solver. The model used here has three fuel enrichment zones. Figure 4a shows the fuel enrichment and Pyrex discrete poison loading in the quarter core. Two other versions of the $2 \mathrm{D}$ core are run with the introduction of control rods into the core. The control rods are grouped into banks to simplify movement. Figure 4 b shows the layout of the control rods and their assigned banks.

The MPACT model, shown in Figure 5a, explicitly models the fuel, burnable poisons, and baffle geometry. The case is run with a $0.05 \mathrm{~cm}$ ray spacing, 16 azimuthal angles, and 2 polar angles per octant using the T-Y quadrature set. The scattering treatment for this case was P2 scattering. The difference in pin powers between the MPACT model and the reference KENO solution is shown in Figure 5b.

Table 1 summarizes the difference in eigenvalue, root-mean square (RMS) difference in pin power, and the maximum difference in pin power for the three different cores. The difference in eigenvalue is reported as percent-milli (pcm) which is the difference multiplied by $10^{5}$. The first is a core with all the control rods removed, the second is a core with Ag-In-Cd (AIC) control 


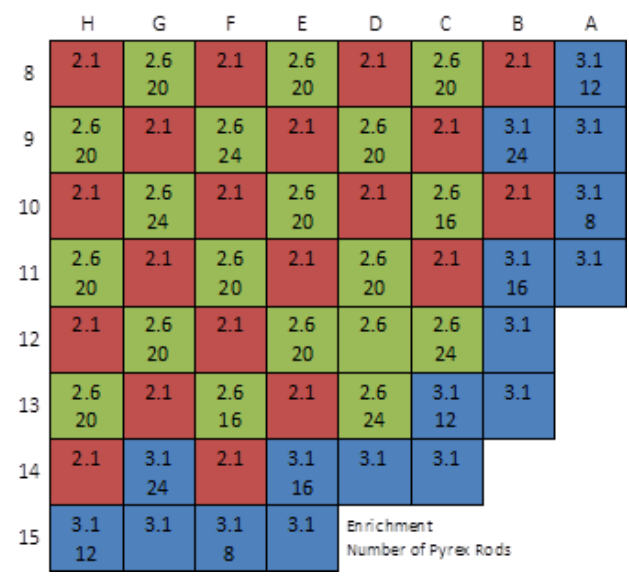

(a) Reactor Core Layout

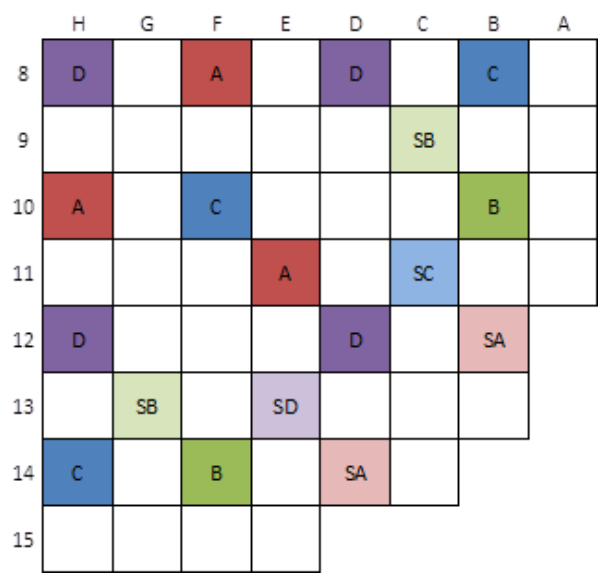

(b) Control Rod Bank Positions

Figure 4: 2D Core Layout (Left) and Control Rod Bank Layout (Right) [28]

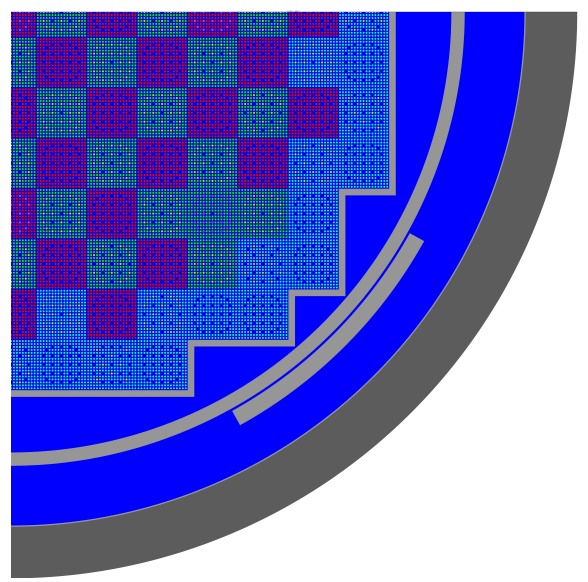

(a) 2D Slice of KENO Model [28]

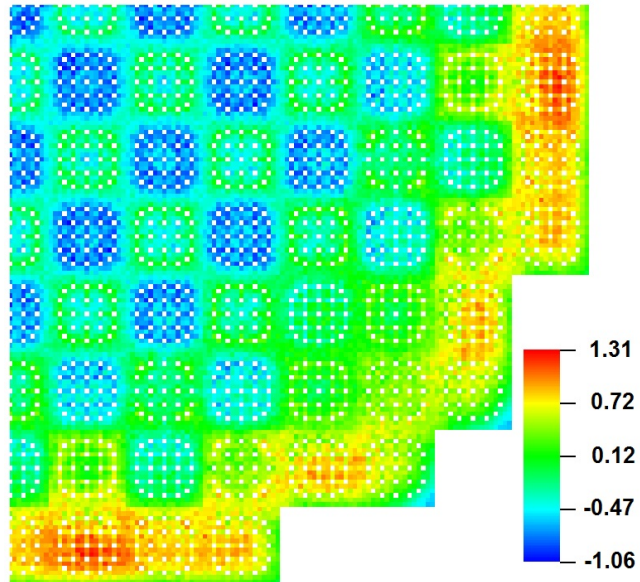

(b) Pin Power Difference

Figure 5: 2D Core Geometry (Left) and Difference in Pin Powers (Right)

rods inserted into the Bank $\mathrm{D}$ positions, and the third has boron carbide $\left(\mathrm{B}_{4} \mathrm{C}\right)$ control rods inserted into Bank D.

For these cases, the KENO stochastic uncertainty is less than $1 \mathrm{pcm}$ for the eigenvalue and the maximum pin power uncertainty is less than $0.15 \%$ difference. In all three cases, the eigenvalue is consistent and close to the KENO calculation. The power distribution shows overall good agreement with a maximum difference less than $1.5 \%$, but there are a few observable 
Table 1: 2D Core Comparisons with KENO

\begin{tabular}{|c|c|c|c|}
\hline Description & k-eff Difference $(\mathrm{pcm})$ & RMS Pin Power & Max Pin Power \\
\hline Unrodded & -100 & $0.50 \%$ & $1.31 \%$ \\
\hline AIC Rodded & -88 & $0.55 \%$ & $1.47 \%$ \\
\hline $\mathrm{B}_{4}$ C Rodded & -87 & $0.53 \%$ & $1.40 \%$ \\
\hline
\end{tabular}

trends. Figure 5b shows a small radial power tilt for the unrodded cases which is slightly exaggerated with the insertion of the control rod. Also, a small bias can be observed between the different batches. Despite these trends, the comparison between MPACT and KENO is good.

\subsection{Single Assembly}

With the accuracy of the 2D MOC solver having been demonstrated, a single 3D assembly is considered. This is used to analyze the accuracy and stability of the 2D/1D method. The 3D assembly, shown in Figure 6, contains many of the major features in the core: top and bottom core plate, top and bottom nozzle, upper plenum, and fuel. The end caps on the fuel pins are included in the input, but MPACT automatically smears them into the coolant to prevent the addition of extra planes in the model. The radial boundary conditions for this problem are reflective and the top and bottom boundary condition is vacuum.

The MPACT model for the single assembly uses the same ray spacing options as before. The major difference in the model is the use of the $\mathrm{S}_{8}$ Gaussian quadrature set in the polar direction. The assembly is decomposed into 60 axial planes, and each plane is given its own MPI processor. MPACT is run using diffusion, $\mathrm{SP}_{3}$, and $\mathrm{SP}_{5}$, and the axial power shape is given in Figure 7. The $\mathrm{SP}_{3}$ and $\mathrm{SP}_{5}$ solutions are nearly identical and indistinguishable in the figure.

A second assembly case is also introduced in the progression problems which has 16 Pyrex rods inserted almost the full length of the assembly. The results for both single-assembly problems are shown in Table 2 for all three axial treatments.

For both of the single assembly cases, the eigenvalue stochastic uncertainty in the reference KENO solution is less than $1 \mathrm{pcm}$, and the maximum pin power uncertainty is less than $0.15 \%$. Figure 7 shows large differences in the diffusion solution at all 7 spacer grid locations. When using the $\mathrm{SP}_{3}$ and $\mathrm{SP}_{5}$ axial solvers, which are capable of capturing the steep gradients in the 


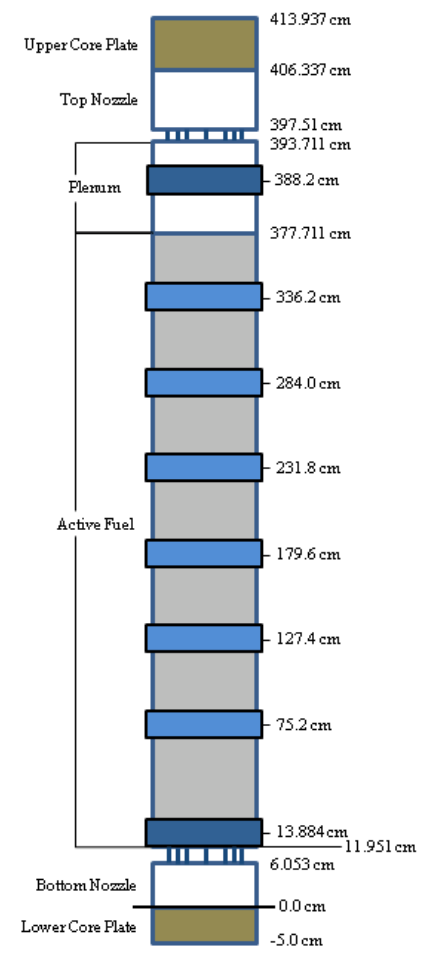

Figure 6: Single Assembly Axial Layout [28]

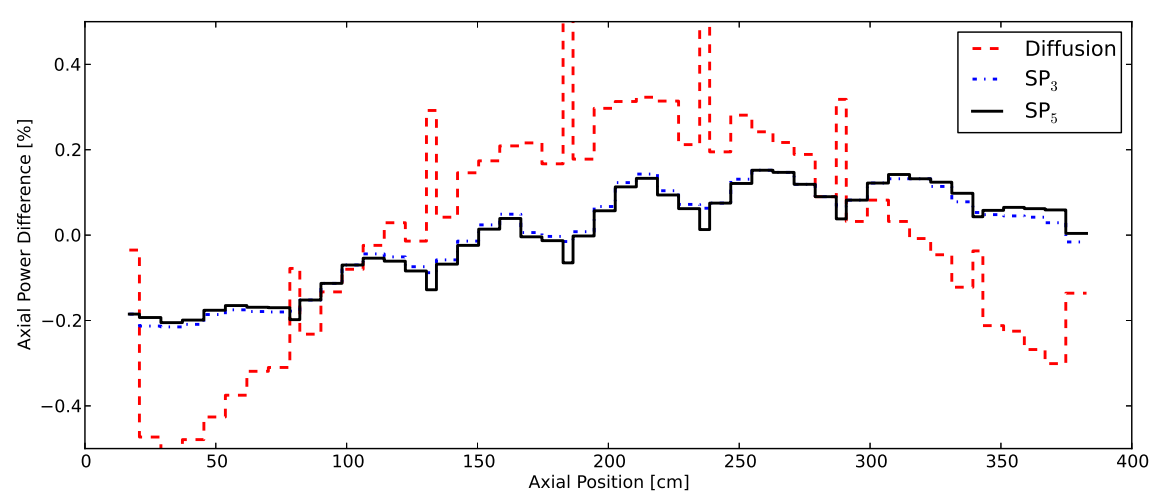

Figure 7: Axial Power Shape for Single Assembly calculation

flux at material boundaries, these differences are significantly reduced. All of the MPACT results show a slight axial tilt, but the $\mathrm{SP}_{3}$ and $\mathrm{SP}_{5}$ solvers also greatly reduce this difference. These improvements are reflected in both 
Table 2: Single Assembly Comparisons with KENO

\begin{tabular}{|c|c|c|c|c|}
\hline Description & Axial Solver & k-eff Difference $(\mathrm{pcm})$ & RMS Pin Power & Max Pin Power \\
\hline \multirow{3}{*}{ No Pyrex } & Diffusion & -123 & $0.28 \%$ & $0.76 \%$ \\
\cline { 2 - 5 } & $\mathrm{SP}_{3}$ & -122 & $0.16 \%$ & $0.43 \%$ \\
\cline { 2 - 5 } & $\mathrm{SP}_{5}$ & -122 & $0.16 \%$ & $0.43 \%$ \\
\hline \multirow{3}{*}{ Pyrex } & Diffusion & -45 & $0.32 \%$ & $1.03 \%$ \\
\cline { 2 - 5 } & $\mathrm{SP}_{3}$ & -57 & $0.17 \%$ & $0.57 \%$ \\
\cline { 2 - 5 } & $\mathrm{SP}_{5}$ & -57 & $0.17 \%$ & $0.56 \%$ \\
\hline
\end{tabular}

the RMS and maximum pin power differences, which are similar to the $2 \mathrm{D}$ core calculation.

\subsection{D Control Cell}

A natural extension of the single assembly problem is the 3D control cell, which considers a 3-by-3 array of assemblies with a control rod cluster inserted into the center assembly. Assemblies adjacent to the rodded assembly have Pyrex inserts with 20 rodlets. This problem creates a challenge for the $2 \mathrm{D} / 1 \mathrm{D}$ methods because of the presence of a strong neutron absorber between axial planes.

The first case considered has the control rod placed at 151 steps withdrawn out of 230 steps. The MPACT model is run with identical settings as used for the single assembly. In this case, the control rod is perfectly aligned with an axial plane boundary to prevent smearing. MPACT is run using diffusion, $\mathrm{SP}_{3}$, and $\mathrm{SP}_{5}$ axial solvers, and the axial power shape compared to the KENO reference solution is given in Figure 8.

Like the single assembly case, the $\mathrm{SP}_{3}$ and $\mathrm{SP}_{5}$ solutions are nearly identical, but the presence of the strong neutron absorber in the control rod creates a large flux gradient that is resolved considerably better with $\mathrm{SP}_{3}$ than with diffusion. Table 3 gives the eigenvalue and pin power comparisons for all three axial solver methods.

Table 3: Control Cell Comparisons with KENO

\begin{tabular}{|c|c|c|c|}
\hline Axial Solver & k-eff Difference $(\mathrm{pcm})$ & RMS Pin Power & Max Pin Power \\
\hline Diffusion & -99 & $0.43 \%$ & $1.44 \%$ \\
\hline $\mathrm{SP}_{3}$ & -97 & $0.29 \%$ & $1.09 \%$ \\
\hline $\mathrm{SP}_{5}$ & -97 & $0.28 \%$ & $1.08 \%$ \\
\hline
\end{tabular}




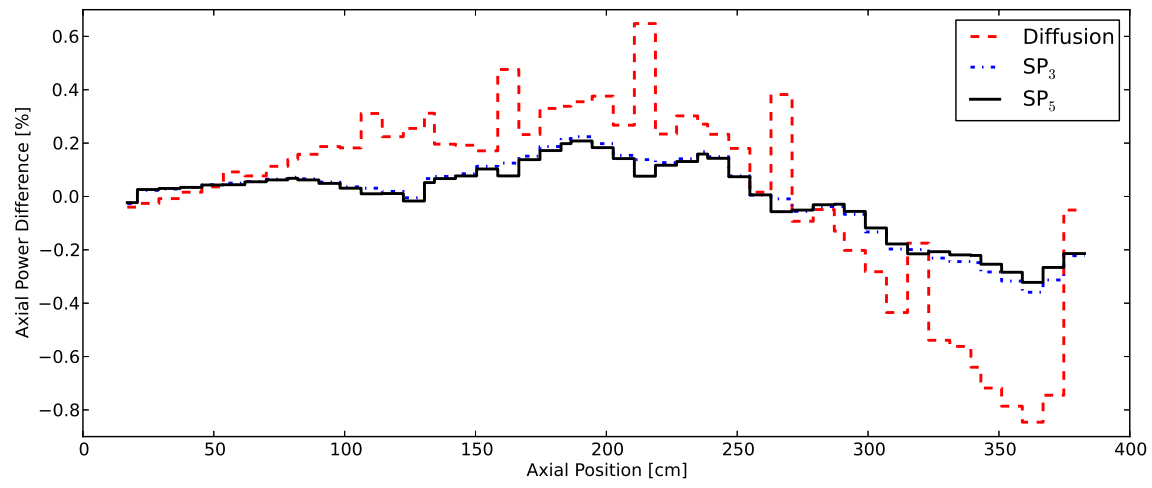

Figure 8: Axial Power Shape for Control Cell calculation

In this case, the reference KENO result has an eigenvalue stochastic uncertainty of less than $0.5 \mathrm{pcm}$, and the maximum pin power uncertainty is less than $0.124 \%$ for all pins whose power is greater than $100 \%$, and less than $0.7 \%$ for the lower-powered pins. As in the assembly problem, the diffusion solution shows significant differences at the spacer grids. There is also a noticeable under-prediction of the power at the top of the core with diffusion. This is caused by the presence of the control rod. The $\mathrm{SP}_{3}$ and $\mathrm{SP}_{5}$ solvers reduce the difference, but a noticeable discrepancy remains where the control rod is inserted.

Next, a series of simulations is performed in which the control rod is withdrawn at 10\% intervals from fully inserted to fully withdrawn. This demonstrates the ability of $2 \mathrm{D} / 1 \mathrm{D}$ to provide high quality data over a large range of control rod positions. For this case, the integral control rod worth,

$$
\rho_{i}=\left(\frac{1}{k_{100 \%}}-\frac{1}{k_{i}}\right) \cdot 10^{5}
$$

where $i$ represents the fraction of the control rod withdrawn, is compared. The reference calculations for these cases, unlike the previous cases, reduce the eigenvalue standard deviation to $1.6 \mathrm{pcm}$, but the pin power statistics are not sufficient to use as a reference. Figure 9 gives the difference in the reference control rod worth to the MPACT results for all three axial solvers.

All three of the MPACT solvers accurately predict the integral control rod worth curve. The $\mathrm{SP}_{3}$ and $\mathrm{SP}_{5}$ solvers do a better job when the control rod is deeply inserted into the model because they capture the strong flux 


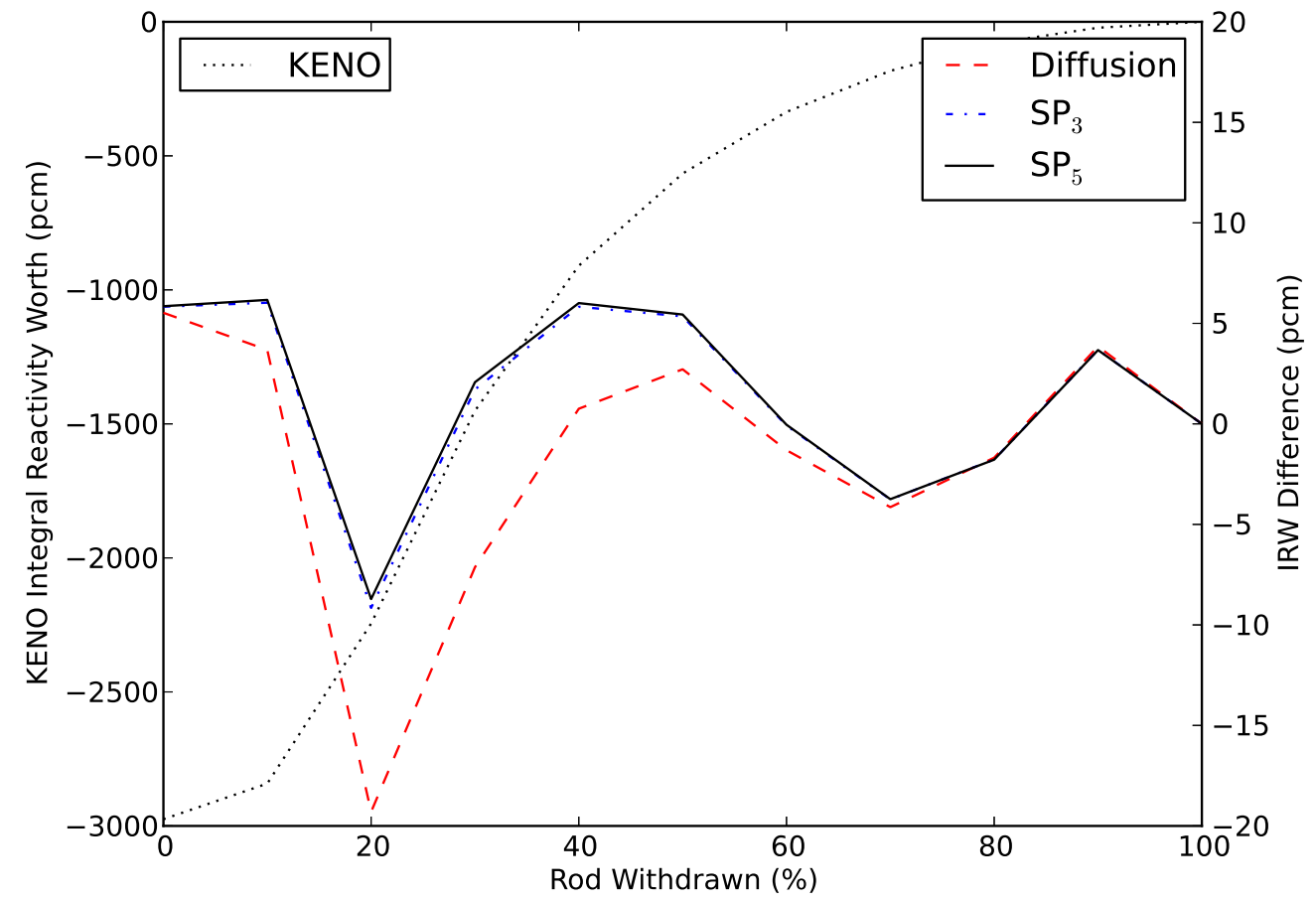

Figure 9: Integral Control Rod Worth for Control Cell calculation

gradients that occur at the rod tip more accurately than diffusion.

\subsection{Full Core}

The $3 \mathrm{D}$ core has many features in common with the $2 \mathrm{D}$ core and single assembly; the same fuel loading pattern is loaded uniformly axially, and the axial detail is the same for all assemblies in the core.

The first comparison made is to a reference KENO case that was run until the statistical deviation of the pin power had an average value of $0.2 \%$. This was done for the initial criticality measurement, where Bank D is withdrawn to 167 steps and the critical boron concentration was measured to be 1285 ppm. Table 4 compares the eigenvalue and pin powers for this case.

For this case, the reference KENO stochastic uncertainty is less than $0.25 \mathrm{pcm}$ for the eigenvalue, and the maximum pin power uncertainty is $0.414 \%$ for the pins with power over $100 \%$ and less than $1.63 \%$ for the lowpower pins. The trends in eigenvalue are similar to the previous cases, but 
Table 4: Full Core Pin Power Comparison

\begin{tabular}{|c|c|c|c|c|}
\hline Description & Axial Solver & k-eff Difference $(\mathrm{pcm})$ & RMS Pin Power & Max Pin Power \\
\hline \multirow{3}{*}{ Initial } & Diffusion & -81 & $0.58 \%$ & $3.46 \%$ \\
\cline { 2 - 5 } & $\mathrm{SP}_{3}$ & -79 & $0.52 \%$ & $2.92 \%$ \\
\cline { 2 - 5 } & $\mathrm{SP}_{5}$ & -79 & $0.52 \%$ & $2.86 \%$ \\
\hline
\end{tabular}

now the pin power differences are slightly elevated. The radial tilt present in the $2 \mathrm{D}$ core is still present, along with the slight axial tilt. These two tilts combine to increase the difference in pin powers to an RMS of $0.52 \%$. The effects of $\mathrm{SP}_{3}$ and $\mathrm{SP}_{5}$ are largely observed in the impact on the maximum pin power difference, reducing it by more than $0.5 \%$.

For the remainder of the cases, KENO is run until the eigenvalue has sufficiently small statistics (less than $1.2 \mathrm{pcm}$ ), but the pin power uncertainty is too large to be used as a reference. Since the $\mathrm{SP}_{3}$ method yields similar results to those of $\mathrm{SP}_{5}$ and requires less computational effort, the remaining cases will use the $\mathrm{SP}_{3}$ axial solver in MPACT. Ten critical conditions which are outlined in Table 5.

Table 5: Full Core Critical Conditions

\begin{tabular}{|c|c|c|c|c|c|c|c|c|c|}
\hline Critical & Boron & \multicolumn{6}{|c|}{ Control Rod Bank (Steps Withdrawn) } \\
\cline { 3 - 10 } Description & $(\mathrm{ppm})$ & SA & SB & SC & SD & A & B & C & D \\
\hline Initial & 1285 & 230 & 230 & 230 & 230 & 230 & 230 & 230 & 167 \\
\hline All Rods Out & 1291 & 230 & 230 & 230 & 230 & 230 & 230 & 230 & 230 \\
\hline Bank SA & 1170 & 0 & 230 & 230 & 230 & 230 & 230 & 230 & 69 \\
\hline Bank SB & 1170 & 230 & 0 & 230 & 230 & 230 & 230 & 230 & 134 \\
\hline Bank SC & 1170 & 230 & 230 & 0 & 230 & 230 & 230 & 230 & 71 \\
\hline Bank SD & 1170 & 230 & 230 & 230 & 0 & 230 & 230 & 230 & 71 \\
\hline Bank A & 1170 & 230 & 230 & 230 & 230 & 0 & 230 & 230 & 97 \\
\hline Bank B & 1170 & 230 & 230 & 230 & 230 & 230 & 0 & 230 & 113 \\
\hline Bank C & 1170 & 230 & 230 & 230 & 230 & 230 & 230 & 0 & 119 \\
\hline Bank D & 1170 & 230 & 230 & 230 & 230 & 230 & 230 & 230 & 18 \\
\hline
\end{tabular}

Both KENO and MPACT are compared to the measured critical eigenvalue in Table 6.

Both KENO and MPACT agree well with the measured multiplication factor $(\mathrm{k}$-eff $=1.0)$. The difference between MPACT and KENO is consistent with all of the previous results considered here, where MPACT tends to 
Table 6: Full Core Criticality Comparisons

\begin{tabular}{|c|c|c|c|c|}
\hline \multirow{2}{*}{$\begin{array}{c}\text { Critical } \\
\text { Description }\end{array}$} & \multicolumn{2}{|c|}{ KENO } & \multicolumn{2}{c|}{ MPACT } \\
\cline { 2 - 5 } & k-eff & Difference $(\mathrm{pcm})$ & k-eff & Difference $(\mathrm{pcm})$ \\
\hline Initial & 0.99990 & -10 & 0.99897 & -103 \\
\hline All Rods Out & 1.00032 & 32 & 0.99940 & -60 \\
\hline Bank SA & 0.99880 & -120 & 0.99804 & -196 \\
\hline Bank SB & 0.99936 & -64 & 0.99837 & -163 \\
\hline Bank SC & 0.99904 & -96 & 0.99802 & -198 \\
\hline Bank SD & 0.99908 & -92 & 0.99805 & -195 \\
\hline Bank A & 0.99902 & -98 & 0.99792 & -208 \\
\hline Bank B & 0.99932 & -68 & 0.99822 & -178 \\
\hline Bank C & 0.99898 & -102 & 0.99804 & -196 \\
\hline Bank D & 0.99898 & -102 & 0.99823 & -177 \\
\hline
\end{tabular}

underestimate the eigenvalue consistently.

\section{Conclusions}

A numerically robust 2D/1D method has been developed for solving the whole-core 3D Boltzmann Transport Equation to calculate fuel pin-resolved neutron fluxes for practical PWR applications. This paper describes the theory of the 2D/1D method and its implementation in the MPACT code, which has become the whole-core deterministic neutron transport solver for the CASL core simulator VERA-CS. Several applications are presented that were performed on industry-class computing clusters. Results are provided that demonstrate the numerical stability and accuracy of the method for a range of practical PWR fuel assembly and full-core applications. Overall, the comparisons with Monte Carlo reference solutions show that both eigenvalue and pin powers can be accurately obtained when using the $\mathrm{SP}_{3}$ and $\mathrm{SP}_{5}$ axial solvers, but larger errors are observed with the diffusion axial solver. It is also observed that the $\mathrm{SP}_{5}$ shows minimal improvement over the $\mathrm{SP}_{3}$ solver. In addition to showing accurate predictions to Monte Carlo reference solutions, MPACT is compared to 10 critical conditions for Watts Bar Nuclear Power Station Unit 1. MPACT produced consistent comparisons with the measured data, having a maximum difference of 208 pcm.

Ongoing work in MPACT will include the coupling of the neutronics to other physics. This will enable the solution of realistic reactor problems at 
power conditions in which there is strong feedback between the neutron transport and the temperature-density distribution. The coupling with MPACT and COBRA-TF [32] is complete, and work is under way to quantify the accuracy of the coupled code models. Further improvements include coupling to other physics, such as fuel materials performance and coolant chemistry. With these features complete, MPACT is capable of providing robust solutions for whole-core pin-resolved power distributions throughout an entire operating cycle overnight, on computing clusters that are currently available to the nuclear industry.

\section{Acknowledgments}

The authors wish to acknowledge the individual members of the MPACT Development Team for their many contributions to the code.

This research was supported by the Consortium for Advanced Simulation of Light Water Reactors (www.casl.gov), an Energy Innovation Hub (http://www.energy.gov/hubs) for Modeling and Simulation of Nuclear Reactors under U.S. Department of Energy Contract No. DE-AC05-00OR22725.

This research used resources of the Oak Ridge Leadership Computing Facility at the Oak Ridge National Laboratory, which is supported by the Office of Science of the U.S. Department of Energy under Contract No. DEAC05-00OR22725.

[1] T. Evans, A. Stafford, R. Slaybaugh, K. Clarno, Denovo: A new threedimensional parallel discrete ordinates code in SCALE, Nuclear Technology 171 (2010) 171-200.

[2] B. Kochunas, A hybrid parallel algorithm for the 3-D method of characteristics solution of the boltzmann transport equation on high performance compute clusters., Ph.D. thesis, University of Michigan (2013).

[3] T. M. Pandya, S. R. Johnson, T. M. Evans, G. G. Davidson, S. P. Hamilton, A. T. Godfrey, Capabilities, implementation, and benchmarking of Shift, a massively parallel monte carlo radiation transport code, Journal of Computational Physics- Companion paper published in same edition of this journal.

[4] K. Smith, J. Rhodes, CASMO-4 characteristics methods for twodimensional PWR and BWR core calculations, Transactions of the American Nuclear Society 83 (2000) 294. 
[5] R. Sanchez, I. Zmijarevic, M. Coste-Delclaux, E. Masiello, S. Santandrea, E. Martinolli, L. Villate, N. Schwartz, N. Guler, APOLLO2: year 2010, Journal of Nuclear Engineering and Technology.

[6] K. Smith, Assembly homogenization techniques for light water reactor analysis, Progress in Nuclear Energy 17 (1986) 303-335.

[7] M. Dahmani, G. Wu, R. Roy, J. Koclas, Development and parallelization of the three-dimensional characteristics solver MCI of DRAGON, in: Proceedings of PHYSOR 2002, Seoul, Korea, 2002.

[8] G. Wu, R. Roy, A new characteristics algorithm for 3D transport calculations, Annals of Nuclear Energy 30 (2003) 1-16.

[9] H. G. Joo, J. Y. Cho, K. S. Kim, C. C. Lee, S. Q. Zee, Methods and performance of a three-dimensional whole-core transport code DeCART, in: PHYSOR 2004 - The Physics of Fuel Cycles and Advanced Nuclear Systems: Global Developments, Chicago, Illinois, 2004.

[10] J. Y. Cho, H. G. Joo, Solution of the C5G7MOX benchmark threedimensional extension problems by the DeCART direct whole core calculation code, Progress in Nuclear Energy 48 (2006) 456-466.

[11] N. Z. Cho, G. S. Lee, C. J. Park, Fusion of method of characteristics and nodal method for 3-d whole core transport calculation, Transactions of the Americal Nuclear Society 86 (2002) 322.

[12] Y. S. Jung, C. B. Shim, C. H. Lim, H. G. Joo, Practical numerical reactor employing direct whole core neutron transport and subchannel thermal/hydraulic solvers, Annals of Nuclear Energy 62 (2013) 357-374.

[13] D. P. Weber, T. Sofu, W. S. Yang, K. S. Kim, T. H. Chun, T. Downar, J. Thomas, H. G. Joo, C. H. Kim, The numerical nuclear reactor: A high fidelity, integrated neutronic, thermal-hydraulic, and thermo-mechanical code, Nuclear Science and Engineering 155 (2007) 395-408.

[14] B. W. Kelley, An investigation of 2D/1D approximations to the 3D boltzmann transport equation, Ph.D. thesis, University of Michigan (2015). 
[15] B. W. Kelley, E. W. Larsen, A consistent 2D/1D approximation to the 3D neutron transport equation", Nuclear Engineering and Design 295 (2015) 598-614.

[16] B. Herman, B. Forget, K. Smith, B. N. Aviles, Improved diffusion coefficients generated from monte carlo codes, in: Proceedings of M\&C 2013, 2013.

[17] K. S. Kim, M. Williams, D. Wiarda, A. Godfrey, Development of a new 47-group library for the casl neutronic simulators, in: Proceedings of M\&C 2015, 2015.

[18] J. Askew, A characteristics formulation of the neutron transport equation in complicated geometries, Tech. Rep. AEEW-R-1108, U.K. Atomic Energy Authority (1972).

[19] B. Collins, B. Kochunas, T. Downar, Assessment of the 2D MOC solver in MPACT, in: Proceedings of M\&C 2013, Sun Valley, ID, 2013.

[20] A. Yamamoto, M. Tabuchi, N. Sugimura, T. Ushio, M. Mori, Derivation of optimum polar angle quadrature set for the method of characteristics based on approximation error for the Bickley function, Journal of Nuclear Science and Technology 44 (2) (2007) 129-136.

[21] H. Finnemann, F. Bennewitz, M. R. Wagner, Interface nodal current technique for multi-dimensional reactor calculation, Atomkernenergie 30 (1977) 123.

[22] R. G. McClarren, Theoretical aspects of the simplified Pn equations, Transport Theory and Statistical Physics 39 (2-4) (2011) 73-109.

[23] S. G. Stimpson, B. Collins, T. Downar, Axial transport solvers for the 2D/1D scheme in MPACT, in: Proceedings of PHYSOR, Kyoto, Japan, 2014 .

[24] K. Smith, Nodal method storage reduction by nonlinear iteration, Transactions of the American Nuclear Society 44 (1983) 265.

[25] T. M. Sutton, B. N. Aviles, Diffusion theory methods for spatial kinetics calculations, Progress in Nuclear Energy 30 (1996) 119. 
[26] B. Kochunas, B. Collins, D. Jabaay, T. J. Downar, W. R. Martin, Overview of development and design of MPACT, in: Proceedings of M\&C 2013, Sun Valley, ID, 2013.

[27] M. Hursin, Full core, heterogeneous, time dependent neutron transport calculations with the 3D code DeCART, Ph.D. thesis, University of California at Berkeley (2010).

[28] A. Godfrey, VERA core physics benchmark progression problem specifications, Tech. Rep. CASL-U-2012-0131-004, CASL, http://www.casl.gov/docs/CASL-U-2012-0131-004.pdf (2014).

[29] F. Franceschini, A. Godfrey, J. Kulesza, R. Oelrich, Westinghouse VERA test stand - zero power physics test simulations for the AP1000 PWR, Tech. Rep. CASL-U-2014-0012-001, CASL, http://www.casl.gov/docs/CASL-U-2014-0012-001.pdf (2014).

[30] S. Goluoglu, L. M. Petrie, M. E. Dunn, D. F. Hollenbach, B. T. Rearden, Monte carlo criticality methods and analysis capabilities in SCALE, Nuclear Technology 174 (2) (2011) 214-235.

[31] SCALE: A comprehensive modeling and simulation suite for nuclear safety analysis and design, Tech. Rep. ORNL/TM-2005/39, Version 6.1, Oak Ridge National Laboratory, available from Radiation Safety Information Computational Center at Oak Ridge National Laboratory as CCC-785 (June 2011).

[32] R. K. Salko, M. N. Avramova, CTF Theory Manual, The Pennsylvania State University. 